\title{
Decapeptide functionalized targeted mesoporous silica nanoparticles with doxorubicin exhibit enhanced apoptotic effect in breast and prostate
} cancer cells

This article was published in the following Dove Press journal:

International Journal of Nanomedicine

\author{
Prajakta Tambe ${ }^{1,2, *}$ \\ Pramod Kumar ${ }^{1,2, *}$ \\ Kishore M Paknikar ${ }^{1,2}$ \\ Virendra Gajbhiye ${ }^{1,2}$ \\ 'Nanobioscience Group, Agharkar \\ Research Institute, Pune, India; \\ ${ }^{2}$ Savitribai Phule Pune University, \\ Pune, India \\ *These authors contributed equally \\ to this work
}

Correspondence: Kishore M Paknikar Nanobioscience Group, Agharkar Research Institute, G. G. Agarkar Road, Pune 4 II 004, India

Tel +9l $2025325 \mid 40$

Email kpaknikar@gmail.com

Virendra Gajbhiye

Nanobioscience Group, Agharkar

Research Institute, G. G. Agarkar Road,

Pune 4I I 004, India

$\mathrm{Tel}+9 \mid 2025325140$

Email cme_virendra@yahoo.co.in

\begin{abstract}
Background: Considering the increase in cancer cases and number of deaths per year worldwide, development of potential therapeutics is imperative. Mesoporous silica nanoparticles (MSNPs) are among the potential nanocarriers having unique properties for drug delivery. Doxorubicin (DOX), being the most commonly used drug, can be efficiently delivered to gonadotropinreleasing hormone $(\mathrm{GnRH})$-overexpressing cancer cells using functionalized MSNPs.
\end{abstract}

Aim: We report the development of decapeptide-conjugated MSNPs loaded with DOX for the targeted drug delivery in breast and prostate cancer cells.

Materials and methods: MSNPs were synthesized and subsequently functionalized with an analog of GnRH by using a heterobifunctional polyethylene glycol as a linker. These targeted MSNPs were then characterized by Fourier transform infrared spectroscopy, scanning electron microscopy, transmission electron microscopy, and Raman spectroscopy. An anticancer drug DOX was loaded and then characterized for drug loading. DOX-loaded nanocarriers were then studied for their cellular uptake using confocal microscopy. The cytotoxicity of DOX-loaded targeted MSNPs and DOX-loaded bare MSNPs was studied by performing MTT assay on MCF-7 (breast cancer) and LNCaP (prostate cancer) cells. Further, acridine orange/ethidium bromide staining, as well as flow cytometry, was performed to confirm the apoptotic mode of cancer cell death.

Results: MSNPs were conjugated with polyethylene glycol as well as an agonist of GnRH and subsequently loaded with DOX. These targeted and bare MSNPs showed excellent porous structure and loading of DOX. Further, higher uptake of DOX-loaded targeted MSNPs was observed as compared to DOX-loaded bare MSNPs in GnRH-overexpressing breast (MCF-7) and prostate $(\mathrm{LNCaP})$ cancer cells. The targeted MSNPs also showed significantly higher $(P<0.001)$ cytotoxicity than DOX-loaded bare MSNPs at different time points. After 48 hours of treatment, the $\mathrm{IC}_{50}$ value for DOX-loaded targeted MSNPs was found to be 0.44 and $0.43 \mu \mathrm{M}$ in MCF-7 and LNCaP cells, respectively. Acridine orange/ethidium bromide staining and flow cytometry analysis further confirmed the pathway of cell death through apoptosis.

Conclusion: This study suggests GnRH analog-conjugated targeted MSNPs can be the suitable and promising approach for targeted drug delivery in all hormone-dependent cancer cells.

Keywords: mesoporous silica nanoparticles, GnRH receptors, receptor-mediated endocytosis, breast and prostate cancer, decapeptide

\section{Introduction}

According to an analysis carried out for the Global Burden of Diseases (2016), cancer is the second leading cause of death worldwide, after cardiovascular ailments. Globally, 
breast cancer is the most prevalent malignancy with 8 million people diagnosed in 2016, followed by prostate cancer (5.7 million). ${ }^{1,2}$ Till date, various chemotherapeutic agents have been employed for treatment of cancer. Doxorubicin (DOX) is one of the effective therapeutic drugs used for a range of solid tumors, namely, carcinomas, sarcomas, and hematological malignancies. ${ }^{3}$ However, cancer treatment using these chemotherapeutic agents is still a challenge owing to several limitations and side effects such as destruction of bone marrow cells, cardiotoxicity, and nephrotoxicity. Moreover, the short biological half-life of DOX demands repetitive dosages, which leads to complications in clinical practice. ${ }^{4}$ Abnormal tumor vasculature also contributes to the complications either by hampering drug delivery to the site of interest or by increasing drug clearance. ${ }^{5}$ These problems of rapid clearance, off-target effects, and inadequate drug delivery can be tackled using nanotechnology-based delivery agents. Nanoparticle (NP)-based carriers, for example, liposomes and polymers, help in prolonging the drug half-life with improved solubility, targeted delivery, sustained drug release, and its accumulation at the tumor site. ${ }^{6}$

Several recent studies indicate that mesoporous silica nanoparticles (MSNPs) are one of the most promising nanocarriers in drug delivery applications. Their structural properties, namely, tunable and large volume pores and vast surface area, make them attractive nanocarriers for drug delivery owing to increased capacity for drug loading. ${ }^{7}$ Furthermore, additive increase in drug loading efficiency is also possible through modification with polyethylene glycol (PEG) moieties, which are also known to increase the circulation time of the particles. These chemically stable, biocompatible MSNPs can also be surface functionalized and modified with stimuli-responsive agents or targeting ligands to ensure controlled release as well as actively targeted drug delivery. ${ }^{8}$ Passive drug delivery, on the other hand, demands high drug dosages for tumor reduction, which is non-economical and beset with toxicity issues. Therefore, targeted delivery is the key solution for reducing the nonspecific drug delivery and adverse effects on normal cells. ${ }^{9}$ Specific targeting of the drug to tumor cells is possible by conjugating antibodies, ${ }^{10,11}$ peptides, ${ }^{12,13}$ or chemical ligands ${ }^{14}$ against the overexpressed receptors present on these cells. It has been reported that $>52 \%$ of breast cancer cells and $86 \%$ of prostate cancer cells overexpress gonadotropin-releasing hormone (GnRH) receptors. ${ }^{15}$ These receptors act by recognizing GnRH (also known as luteinizing hormone-releasing hormone), which exerts a stimulatory effect on gonadotropin secretion, leading to their overexpression on cancer cells. ${ }^{16}$ Thus, synthetically designed GnRH agonists can act as good candidates for targeting cancer cells bearing these highly overexpressed receptors. Triptorelin (pGlu1-His2-Trp3-Ser4-Tyr5-DTrp6-Leu7-Arg8-Pro9-Gly10- $\mathrm{NH}_{2}$ ) is one such agonist of native, ${ }^{17}$ synthetic GnRH that can be potentially employed for targeted drug delivery. ${ }^{18}$ In a novel approach, we have recently reported the use of peptide-conjugated dendritic nanocarrier for targeted siRNA delivery and gene silencing in breast as well as prostate cancer cells. Gene silencing studies in luciferase-expressing cancer cells demonstrated that the ligand-conjugated targeted NPs exhibited extremely significant silencing of luciferase gene than non-targeted NPs. Thus, the study suggested that conjugating peptide could be a promising approach for targeted gene silencing in luteinizing hormone-releasing hormone-overexpressing cancer cells. ${ }^{19}$

Herein, we have made an attempt to develop triptorelinconjugated MSNP nanocarriers, which would specifically target the anticancer drug to GnRH receptor-overexpressing tumor cells. Briefly, PEG and triptorelin ligand-conjugated MSNPs were synthesized (MSNP-PEG-triptorelin) and characterized. They were loaded with an anticancer drug, DOX. These DOX-loaded MSNPs were studied for their uptake in the breast (MCF-7) and prostate (LNCaP) cancer cell lines. Further, the cytotoxicity of DOX-loaded targeted MSNPs, DOX-loaded bare MSNPs, and free DOX was evaluated at different concentrations in both the cancer cell lines. Also, the mode of cytotoxicity was assessed to confirm apoptosis in the cancer cells. Overall, the results obtained suggest that the developed multifunctional targeted nanocarriers could be used in targeted therapy of various cancers.

\section{Materials and methods Materials}

Tetraethylorthosilicate (TEOS), cetyltrimethylammonium chloride (CTAC), triethanolamine, $N, N^{\prime}$-dicyclohexylcarbodiimide, 4-dimethylaminopyridine, Hoescht 33342, ethidium bromide (EB), acridine orange (AO), and solvents, namely, methanol and dimethylsulfoxide (DMSO), were purchased from Sigma-Aldrich (Bangalore, India). Cell culture media, namely, RPMI-1640 and DMEM, were purchased from Thermo Fisher Scientific (Chennai, India). The FBS used in cell culture was purchased from HIMEDIA ${ }^{\circledR}$ (Mumbai, India). All chemicals used were of analytical grade.

\section{Synthesis of MSNPs}

MSNPs were synthesized using the previously reported method by our group. ${ }^{20}$ This method involves the use of 
CTAC as a template, triethanolamine as catalyst, and TEOS as a precursor. In brief, distilled water $(100 \mathrm{~mL})$ was heated up to a temperature of $95^{\circ} \mathrm{C}$ and then CTAC $(18.75 \mathrm{mmol})$ along with triethanolamine $(3.76 \mathrm{mmol})$ was added. Further, TEOS (13.43 mmol) was added to the reaction mixture and stirred for 2 hours. After cooling down to room temperature (RT), the reaction mixture was centrifuged at $14,400 \times g$ for 1 hour. The pellet was then dispersed in methanol and again centrifuged at $14,400 \times g$ for 40 minutes at RT. The MSNPs pellet was washed with methanol followed by water, and then, extraction of CTAC was carried out in acidic methanol. The reaction mixture was refluxed for 24 hours and washed with methanol followed by water. The synthesized MSNPs were lyophilized, stored at RT, and used in further studies.

\section{Synthesis of targeted (MSNP-PEG- triptorelin) MSNPs}

Targeted MSNPs (MSNP-PEG-triptorelin) were prepared by conjugating PEG and triptorelin to the surface of MSNPs. First, triptorelin $(0.07 \mathrm{nmol})$ was mixed with a heterobifunctional PEG (NHS-PEG-COOH, $0.07 \mathrm{nmol}$ ) in DMSO and stirred for 24 hours (Scheme 1). After 24 hours stirring, $N, N^{\prime}$-dicyclohexylcarbodiimide (1 equivalent) and 4-dimethylaminopyridine ( $7 \mathrm{~mol} \%$ ) were added to the reaction mixture at $0^{\circ} \mathrm{C}$ and stirred at $\mathrm{RT}$ for 1 hour. Then, MSNPs $(10 \mathrm{mg})$ were added to the mixture and allowed to react for 24 hours. The reacted mixture was centrifuged and the pellet was washed thrice with deionized water to remove any unreacted reagents and by-products. The washed pellet was lyophilized for future use.

\section{Drug loading}

The anticancer drug DOX was loaded on the bare as well as developed targeted MSNPs and further studies were carried out for their specific delivery into the cancer cells. For drug loading, DOX and targeted MSNPs were admixed in the ratio of $1: 1(\mathrm{w} / \mathrm{w})$ in distilled water and the mixture was stirred for 48 hours at RT. The reaction mixture was centrifuged, and the obtained MSNPs pellet was washed with distilled water to remove unloaded DOX followed by lyophilization. A similar method was followed for drug loading in bare MSNPs. The unreacted DOX in the supernatant was estimated using ultraviolet-visible spectroscopy (at $480 \mathrm{~nm}$ wavelength), and the percent weight of DOX loaded per milligram of MSNPs was calculated by using the following formula:

$$
\% \text { Loading capacity }=\frac{\text { Entrapped DOX }}{\text { MSNPs weight }} \times 100
$$

DOX loading on the MSNPs was confirmed by Fourier transform infrared (FT-IR) spectroscopy.

\section{Drug release study}

The release profile of the DOX-loaded targeted MSNPs was studied at two different $\mathrm{pH}$ values $(\mathrm{pH} 7.4$ and 5 [acetate buffer]) to simulate the physiological and cancer environment. DOX-loaded targeted MSNPs were suspended in acetate buffer $(\mathrm{pH} 5)$ or PBS ( $\mathrm{pH} 7.4)$. These suspensions were then dialyzed against $50 \mathrm{~mL}$ PBS using $1 \mathrm{kDa}$ dialysis bags. At predetermined time points, $1 \mathrm{~mL}$ of solution was removed and replenished with $1 \mathrm{~mL}$ of fresh release medium. The amount of DOX released was determined by ultravioletvisible spectroscopy.

\section{Characterization}

The synthesized MSNPs were characterized for size and zeta potential using Nanosight, LM10 (Malvern Instruments, Malvern, UK) and DelsaNano (Beckman Coulter, Brea, CA, USA), respectively. Surface morphology of MSNPs and their porosity were studied by field emission-scanning electron microscopy (Bruker, Fällanden, Switzerland) and transmission electron microscopy (TEM, operated at $200 \mathrm{kV}$ accelerating voltage; Bruker), respectively. For TEM, MSNPs were deposited on carbon-coated copper TEM grids of 200 mesh and air dried. Further, targeted and bare MSNPs were characterized using FT-IR spectroscopy by dispersing the samples in $\mathrm{KBr}$ pellets. The FT-IR spectra were recorded in the range of $400-4,000 \mathrm{~cm}^{-1}$. Additionally, Raman spectroscopy was also performed for bare and targeted MSNPs to further confirm the conjugation. For this, concentrated solutions of bare, as well as targeted MSNPs were placed on glass slides and allowed to dry for making a layer of MSNPs. Then, separate Raman spectra were recorded for targeted and bare MSNPs at RT between 3,600 and $100 \mathrm{~cm}^{-1}$.

\section{Cell culture}

GnRH receptor-overexpressing human mammary adenocarcinoma (MCF-7) and prostate cancer ( $\mathrm{LNCaP}$ ) cell lines were obtained from the National Center for Cell Science (Pune, India). Cell culture conditions were maintained according to the requirement of the respective cell lines. The MCF-7 cell line was maintained in DMEM with 10\% FBS (Himedia) and $1 \%$ penicillin/streptomycin (200 units of penicillin and $200 \mu \mathrm{g}$ of streptomycin sulfate per milliliter of DMEM; Himedia). LNCaP cell line was maintained at $37^{\circ} \mathrm{C}$ in a humidified $\mathrm{CO}_{2}(5 \%)$ incubator using RPMI-1640 media supplemented with $10 \%$ FBS and penicillin/streptomycin. 


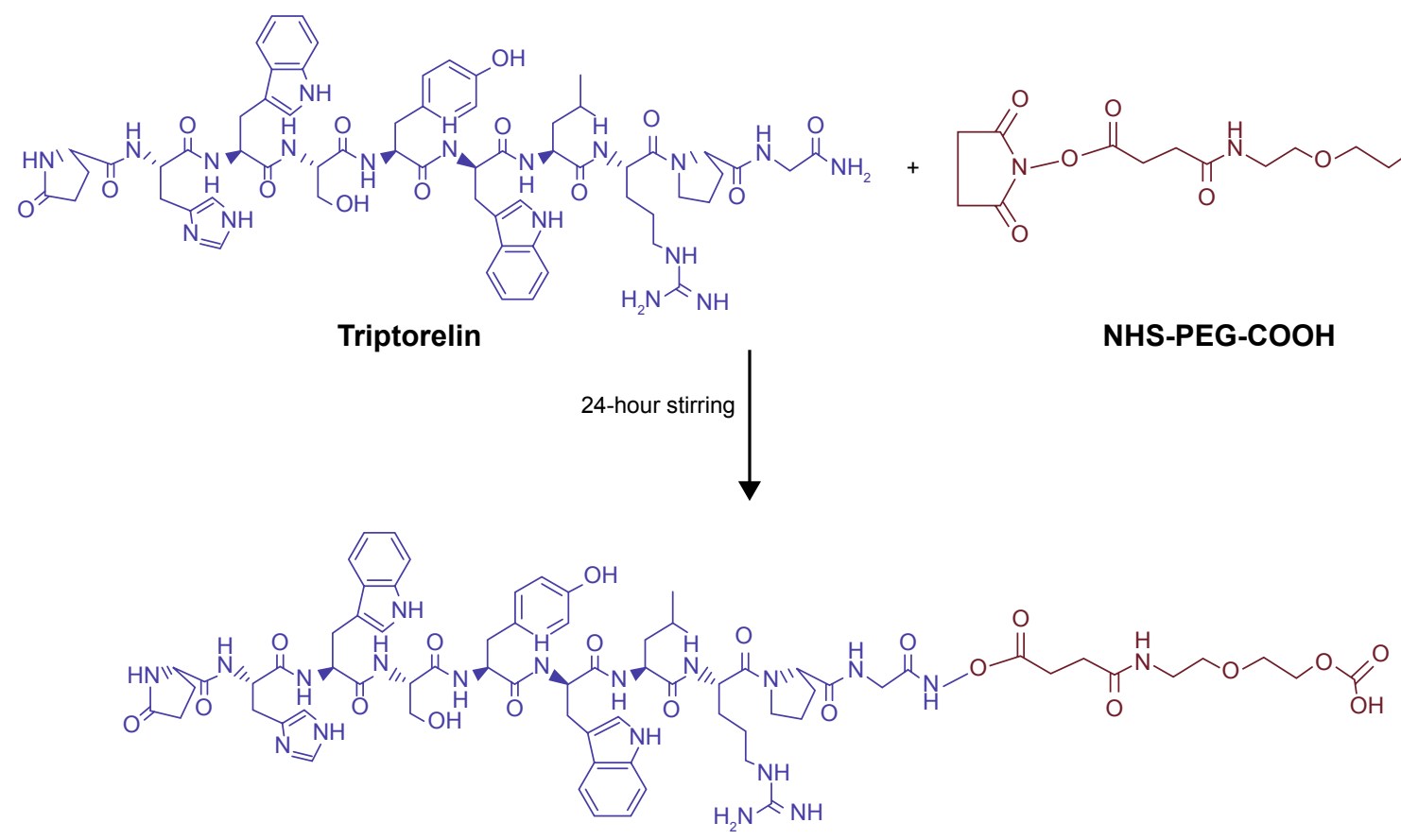

Triptorelin-PEG-COOH
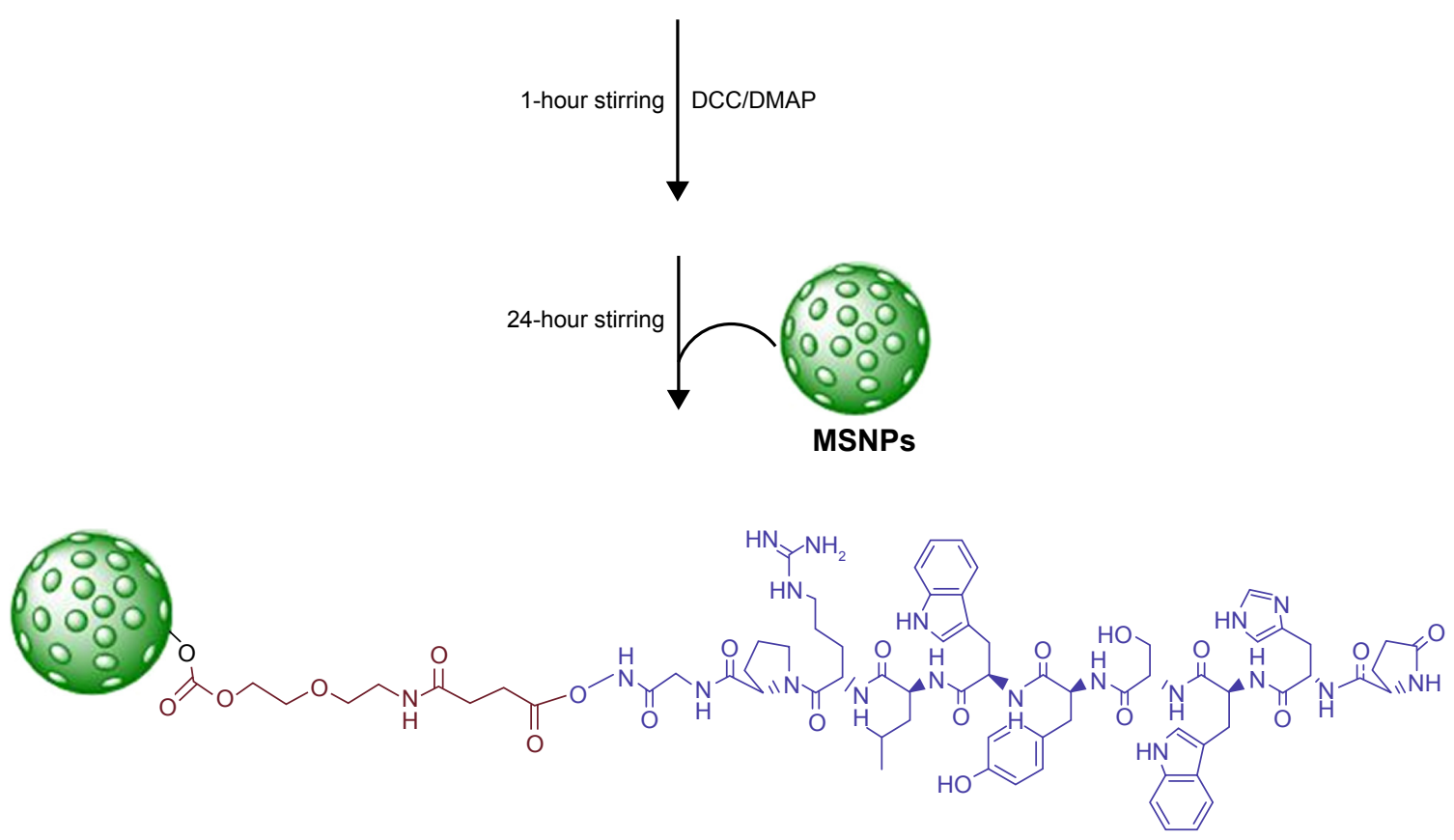

Targeted MSNPs (MSNP-PEG-triptorelin)

Scheme I Schematic representation of the synthesis for targeted MSNPs.

Abbreviations: DCC, N, N'-dicyclohexylcarbodiimide; DMAP, 4-dimethylaminopyridine; MSNPs, mesoporous silica nanoparticles; PEG, polyethylene glycol.

\section{Cellular internalization}

The uptake of DOX-loaded and ligand-conjugated MSNPs in cancer cells was studied using confocal microscopy. The MCF-7 and LNCaP cells were seeded at a density of $1 \times 10^{5}$ cells per well and grown for 24 hours on coverslips. Following 24 hours of incubation, the cells were treated with
DOX-loaded targeted MSNPs, DOX-loaded bare MSNPs (containing $10 \mu \mathrm{g}$ of DOX), and free DOX $(10 \mu \mathrm{g})$ for 2 hours in serum-free media at $37^{\circ} \mathrm{C}$. After incubation, the cells were washed with PBS, fixed, and permeabilized with $4 \%$ paraformaldehyde and $0.2 \%$ Triton-X. The cells were further stained with Hoechst 33342 (for nucleus) and then 
imaged using a confocal microscope (Leica Microsystems, Wetzlar, Germany).

\section{Cytotoxicity studies}

Cytotoxicity of free DOX as well as DOX-loaded targeted MSNPs and DOX-loaded bare MSNPs was assessed on both the cancer cells by determining their viability using MTT assay. For this, MCF-7 and LNCaP cells were seeded at a density of $1 \times 10^{4}$ in 96 -well plate cells for 24 hours at $37^{\circ} \mathrm{C}$ with $5 \% \mathrm{CO}_{2}$. The cells were treated with free DOX, DOXloaded targeted MSNPs, and DOX-loaded bare MSNPs (with effective DOX concentrations of $0.01,0.1,1$, and $10 \mu \mathrm{M}$ ) for 24 and 48 hours in serum-free media. Twenty-four hours posttreatment, $20 \mu \mathrm{L}$ of MTT reagent $(5 \mathrm{mg} / \mathrm{mL})$ was added to each well and incubated at $37^{\circ} \mathrm{C}$ for 4 hours. Subsequently, the formazan crystals were solubilized by the addition of DMSO $(200 \mu \mathrm{L})$ and absorbance was measured at $570 \mathrm{~nm}$ on BioTek Synergy Instrument.

\section{Apoptosis studies in cancer cells}

DOX-induced apoptosis in cancer cells was studied using $\mathrm{AO} / \mathrm{EB}$ double staining assay. Both MCF-7 and LNCaP cells were seeded at a density of $1 \times 10^{5}$ cells per well. Following overnight growth, cells were treated with free DOX, DOXloaded targeted MSNPs, and DOX-loaded bare MSNPs for the next 24 hours. The cells were then washed with PBS, stained with $\mathrm{AO} / \mathrm{EB}(100 \mu \mathrm{g} / \mathrm{mL})$ for 5 minutes, and further examined under a fluorescence microscope. ${ }^{21}$

Apoptosis was also confirmed quantitatively using flow cytometry analysis by staining the cells with Annexin V-fluorescein isothiocyanate (FITC) and propidium iodide (PI; Thermo Fisher Scientific). Briefly, MCF-7 cells were seeded at a density of $1 \times 10^{5}$ per well and grown for 24 hours. Next day, the cells were treated with $10 \mu \mathrm{M}$ free DOX as well as DOX-loaded targeted NPs and DOX-loaded bare NPs containing equivalent quantity of DOX $(10 \mu \mathrm{M})$ separately in serum-free media and then incubated for 12 hours. After 12 hours, the cells were harvested, washed with PBS, and then subjected to centrifugation. The pellet was resuspended in $1 \mathrm{X}$ Annexin binding buffer and then subsequently stained with PI (necrotic cells) and Annexin V-FITC (live/apoptotic cells). After incubating for 15 minutes with the stains, the cells were analyzed by flow cytometer (Attune NxT; Thermo Fisher Scientific).

\section{Statistical analyses}

All experiments were performed in triplicate and data were expressed as mean $\pm \mathrm{SD}$. The mean values of groups were compared by two-way ANOVA with Bonferroni's multiple comparison tests. The GraphPad Prism software was used to perform the statistical analyses. $P$-value $<0.05$ was considered as statistically significant.

\section{Results}

\section{Synthesis and characterization}

Decapeptide-conjugated targeted MSNPs were synthesized and evaluated for their ability to deliver DOX into two different cancer cells. MSNPs were successfully synthesized and characterized for their size, zeta potential, and morphology. The hydrodynamic size of the MSNPs was found to be $113 \pm 34 \mathrm{~nm}$ by Nanosight (Figure 1A), while the hydrodynamic size was found to be $55 \mathrm{~nm}$ by TEM. Further, the surface charge was determined to be $-24.39 \pm 3.2 \mathrm{mV}$. Upon conjugation of PEG-triptorelin on MSNPs surface, the size observed in dynamic light scattering was $159 \pm 50 \mathrm{~nm}$ (Figure 1B) with a surface charge of $-17.94 \pm 1.89 \mathrm{mV}$. The synthesized MSNPs showed porous architecture as observed in TEM (Figure 1C), and SEM analysis showed spherical morphology (Figure 1D). The FT-IR spectrum of MSNPs showed absorption peaks at 1,091 and 3,432 $\mathrm{cm}^{-1}$ demonstrating $\mathrm{Si}-\mathrm{O}-\mathrm{Si}$ asymmetric stretching vibrations and $\mathrm{Si}-\mathrm{OH}$ stretching vibrations (Figure S1). ${ }^{22}$ The conjugation of PEG-triptorelin on the surface of MSNPs was confirmed by the peak at $1,684 \mathrm{~cm}^{-1}$ $\left(1,680-1,630 \mathrm{~cm}^{-1}\right)$, which corresponds to $\mathrm{C}=\mathrm{O} .{ }^{23}$ Also, the peak at $1,628 \mathrm{~cm}^{-1}$ in MSNPs spectra was replaced by a peak at 1,635.64 $\mathrm{cm}^{-1}$ in targeted MSNPs, which is related to the $\mathrm{N}-\mathrm{H}$ bond of the amide group. Additionally, Raman spectroscopy was performed to further validate the conjugation of triptorelin on MSNPs. Spectra of targeted MSNPs showed a signal at $2,935.39 \mathrm{~cm}^{-1}$ for the amide group of triptorelin (Figure S2). ${ }^{24}$

\section{Drug loading and its characterization}

Drug loading on the targeted and bare MSNPs was performed by stirring DOX and MSNPs continuously at a w/w ratio of $1: 1$. The concentrations of DOX used and subsequently detected in the supernatant (unentrapped DOX) were used to calculate drug loading on the MSNPs. Results demonstrated $51.66 \pm 2.88$ and $40.67 \pm 3.78 \mathrm{wt} \%$ drug loading in targeted and bare MSNPs, respectively. In FT-IR spectroscopy, the characteristic absorption peak at $1,639 \mathrm{~cm}^{-1}$ was observed for DOX-loaded MSNPs, which could be attributed to the overlap of peaks at $1,607 \mathrm{~cm}^{-1}$ (pure DOX) and 1,628 $\mathrm{cm}^{-1}$ (MSNPs-PEG-triptorelin). Further, an increase in the intensity of the broad peak at $3,550 \mathrm{~cm}^{-1}$ was observed due to additional hydroxyl groups introduced by DOX, which confirmed successful loading on the MSNPs (Figure S3). 

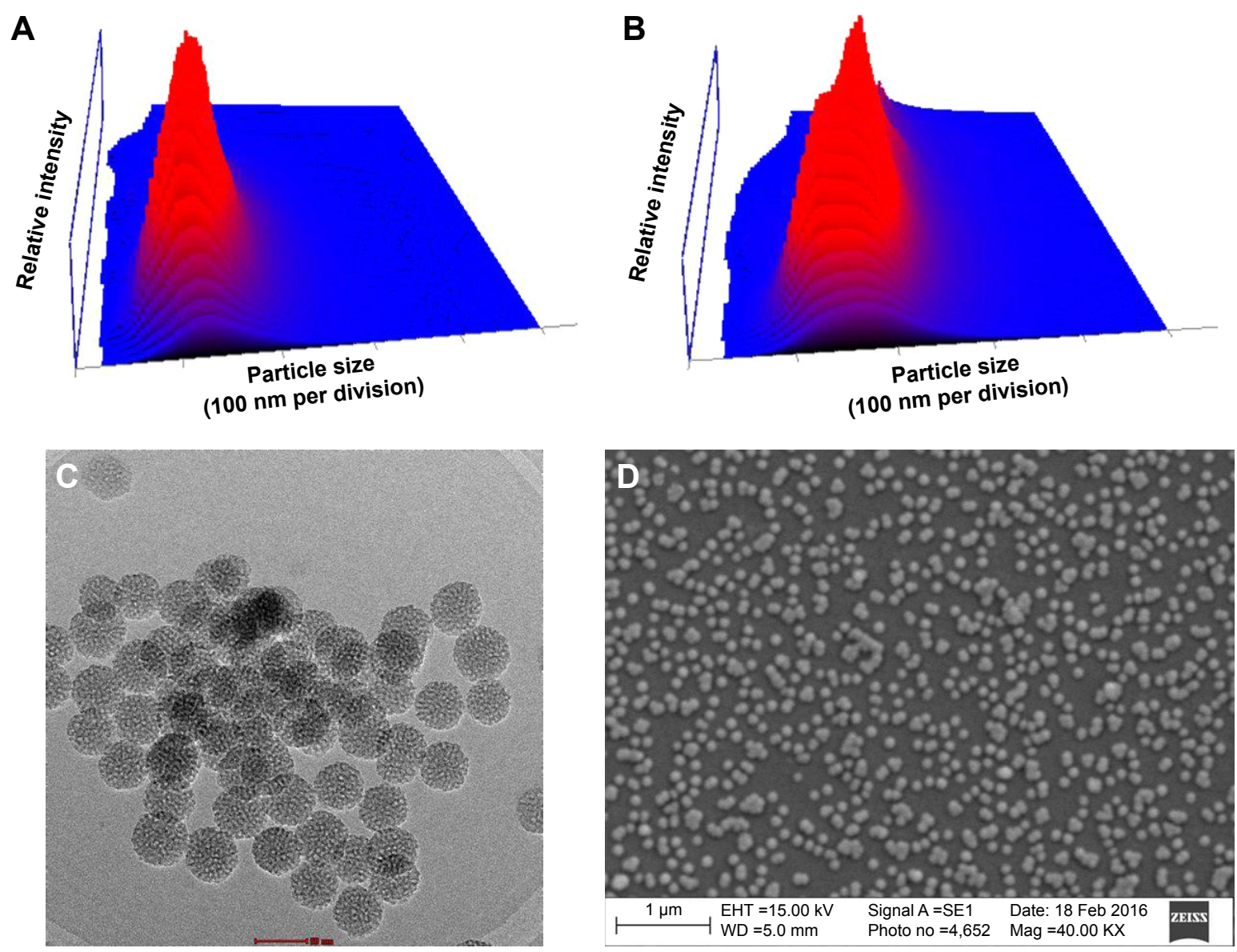

Figure I Characterization studies.

Notes: Particle size of (A) bare MSNPs (B) targeted MSNPs. (C, D) TEM and SEM images of bare MSNPs.

Abbreviations: EHT, electron high tension; Mag, magnification; MSNPs, mesoporous silica nanoparticles; SEI, secondary electrons I; SEM, scanning electron microscopy; TEM, transmission electron microscopy; WD, working distance.

\section{Drug release study}

For utilizing nanocarriers in cancer therapy, an adequate understanding of tumor physiology is imperative to safeguard tumor site delivery of nanocarriers with minimal drug leakage. Nanocarriers with the ability to discharge drug at acidic $\mathrm{pH}$ while retaining the same cargo at physiological $\mathrm{pH}$ are preferred since the tumor sites have slightly acidic $\mathrm{pH}$ (5-6.5). These $\mathrm{pH}$-responsive nanocarriers could have a significant impact on the controlled delivery of cargo. The release profile of MSNP-PEG-triptorelin (Figure S4) showed $\mathrm{pH}$-dependent drug release with an initial burst release of DOX at both $\mathrm{pHs}$ followed by a sustained release over the time period of the study. At pH 7.4, 49.52\% of DOX release was observed in 96 hours. On the other hand, $96.4 \%$ of DOX release was observed in 96 hours at $\mathrm{pH} 5$.

\section{Cellular internalization}

After successful synthesis and characterization of the nanocarriers, cellular internalization studies were performed.
The MCF-7 and LNCaP cells were treated with DOX-loaded bare and targeted MSNPs as well as free DOX for 2 hours at a particular concentration and then imaged using a confocal microscope. Nuclei were stained with Hoechst 33342, while the internalization of DOX-loaded MSNPs was visualized by the fluorescent property of DOX (Figure 2). ${ }^{25}$ Confocal images displayed significantly higher uptake of DOX-loaded targeted MSNPs (Video S1) as compared to DOX-loaded bare MSNPs (Video S2) in MCF-7 cells (Figure 2A). Free DOX was seen entering the MCF-7 as well as LNCaP cells and it accumulated in the nuclei, which appeared red (Videos $\mathrm{S} 3$ and $\underline{\mathrm{S}} 4$, respectively). Similar results were observed in $\mathrm{LNCaP}$ cells (Figure 2B) wherein DOX-loaded targeted MSNPs showed higher internalization (Video S5) as compared to DOXloaded bare MSNPs (Video S6). These results were also quantified by calculating the intensity of internalized DOX in the cells using ImageJ software (Figure S5). Additionally, 3D Z-stack images of MCF-7 cells treated with DOX-loaded targeted MSNPs (Figure 3A) and DOX-loaded bare MSNPs 

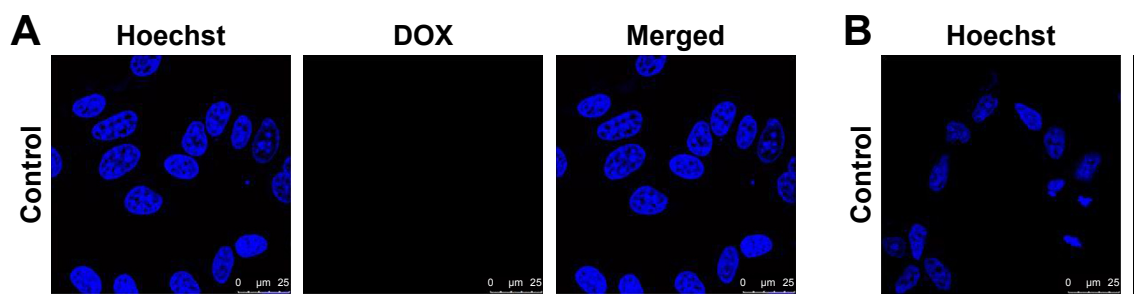

DOX

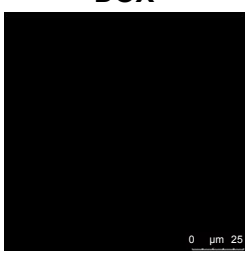

Merged
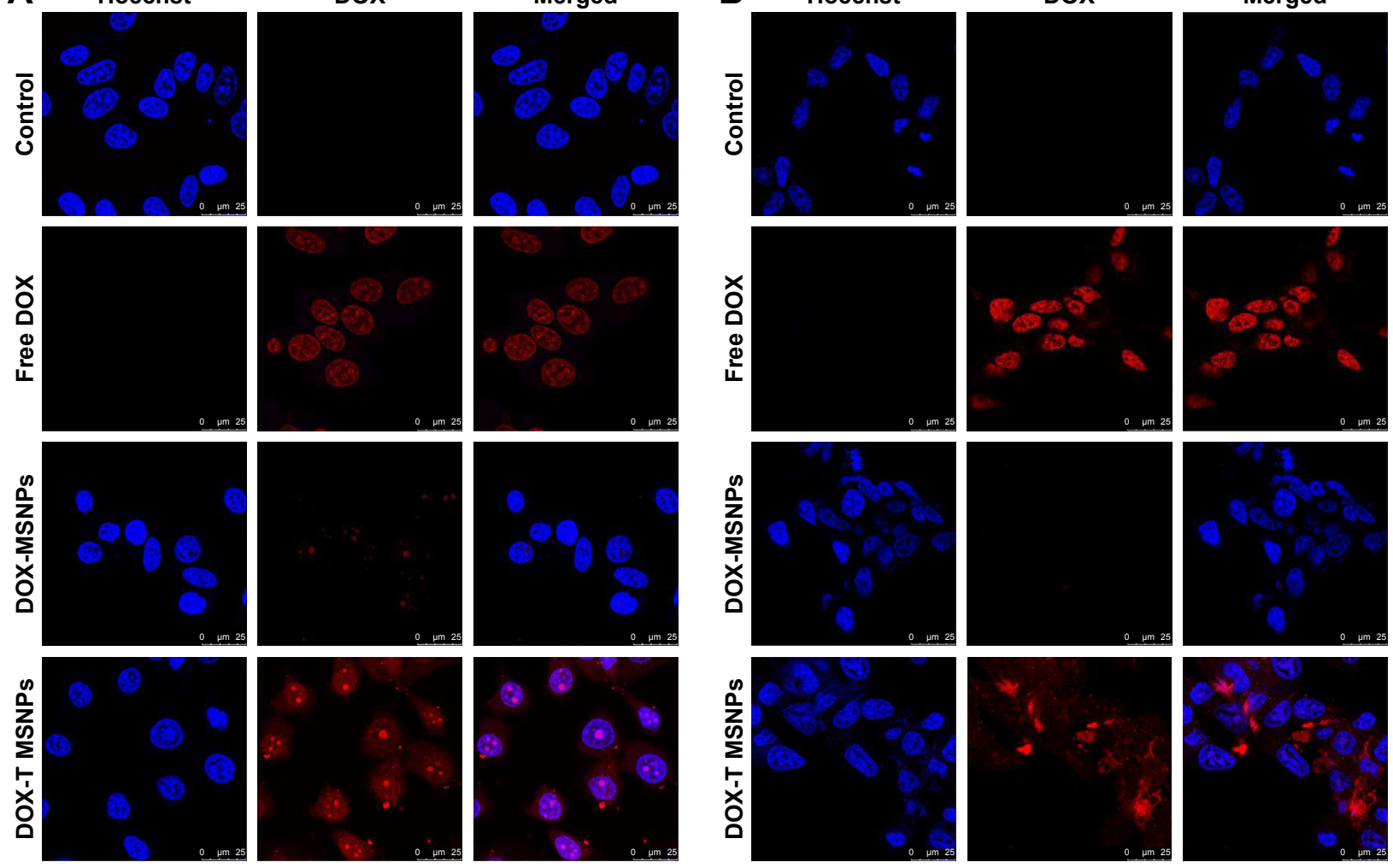

Figure 2 Cellular internalization.

Note: Confocal images of DOX-loaded bare (DOX-MSNPs) and DOX-loaded targeted MSNPs (DOX-T MSNPs) in (A) MCF-7 and (B) LNCaP cells.

Abbreviations: DOX, doxorubicin; MSNPs, mesoporous silica nanoparticles.

(Figure 3B) further confirmed their internalization in the cytoplasm. Similarly, internalization of DOX-loaded targeted MSNPs and DOX-loaded bare MSNPs (Figure 3D) in the cytoplasm of LNCaP cells was also confirmed by $3 \mathrm{D}$ Z-stack images (Figure 3C and D, respectively).

\section{Cytotoxicity studies}

Cytotoxicity studies were performed to determine the anticancer activity of the DOX-loaded MSNPs on the cancer cells by estimating percent viability. Cells were incubated with DOX-loaded targeted MSNPs, DOX-loaded bare MSNPs,

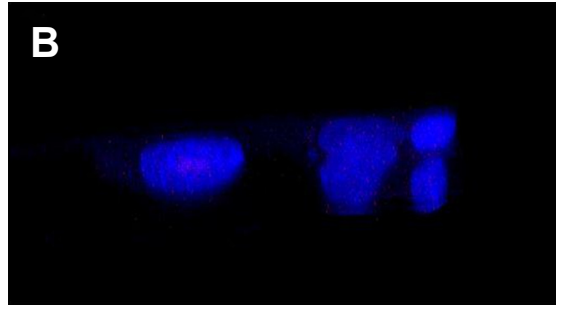

D

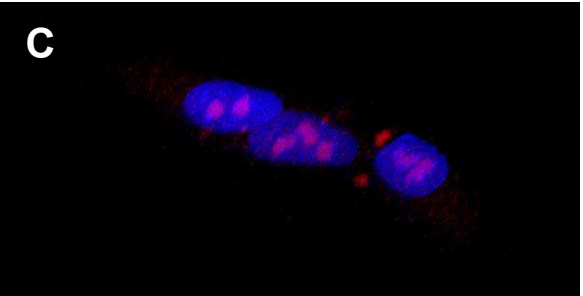

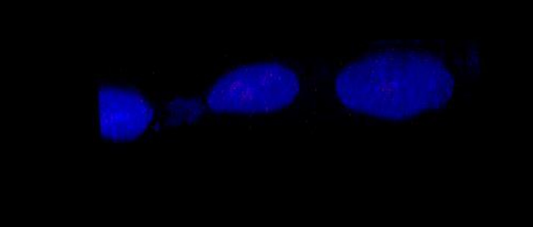

Figure 3 Cellular internalization by Z-stack imaging.

Note: Z-stack images of (A, B) DOX-loaded targeted and bare MSNPs in MCF-7 cells, respectively, and (C, D) DOX-loaded targeted and bare MSNPs in LNCaP cells, respectively. Abbreviations: DOX, doxorubicin; MSNPs, mesoporous silica nanoparticles. 
and free DOX for 24 and 48 hours. After incubation, MTT assay was performed wherein MCF-7 cells treated with DOXloaded targeted MSNPs showed very significant $(P<0.05)$ toxicity at $10 \mu \mathrm{M}$ concentration at 24 hours. Nevertheless, further higher and extremely significant $(P<0.001)$ cytotoxicity was observed at 48 hours for targeted MSNPs than for DOX-loaded bare MSNPs and free DOX (Figure 4A and B). Similar results were obtained in LNCaP cells wherein the DOX-loaded targeted MSNPs displayed extremely significant $(P<0.001)$ cytotoxicity as compared to free DOX and DOX-loaded bare MSNPs (Figure 4C and D). The results obtained in the MTT assay corroborated well with the internalization data. The percentage viability in MCF-7 cells at $10 \mu \mathrm{M}$ concentration after 48 hours treatment was observed to be $54.5 \% \pm 2.8 \%, 34.6 \% \pm 2.76 \%$, and $46.8 \% \pm 1.82 \%$ for free DOX, DOX-loaded targeted MSNPs, and DOX-loaded bare MSNPs, respectively. Similarly, in LNCaP cells, after 48 hours treatment, $35.7 \% \pm 4.7 \%, 27.9 \% \pm 5.8 \%$, and
$44.7 \% \pm 4.2 \%$ cell viability was observed for free DOX, DOXloaded targeted MSNPs, and DOX-loaded bare MSNPs, respectively.

The $\mathrm{IC}_{50}$ values for DOX-loaded targeted MSNPs and DOX-loaded bare MSNPs were found to be 0.44 and $0.65 \mu \mathrm{M}$, respectively, in MCF-7 cells. In LNCaP cells, the $\mathrm{IC}_{50}$ values were 0.43 and $1.082 \mu \mathrm{M}$ for DOX-loaded targeted MSNPs and DOX-loaded bare MSNPs, respectively. The decrease in cell viability, that is, enhanced activity of DOX-loaded targeted MSNPs was clearly due to triptorelin ligand conjugation.

\section{Apoptosis studies in cancer cells}

DOX induces apoptosis in various cancers by intercalating in the DNA of the cells. Herein, we have carried out AO/ EB double staining to determine the apoptotic effect of DOX delivered in the breast and prostate cancer cells. The images demonstrated that MCF-7 cells treated with only DOX
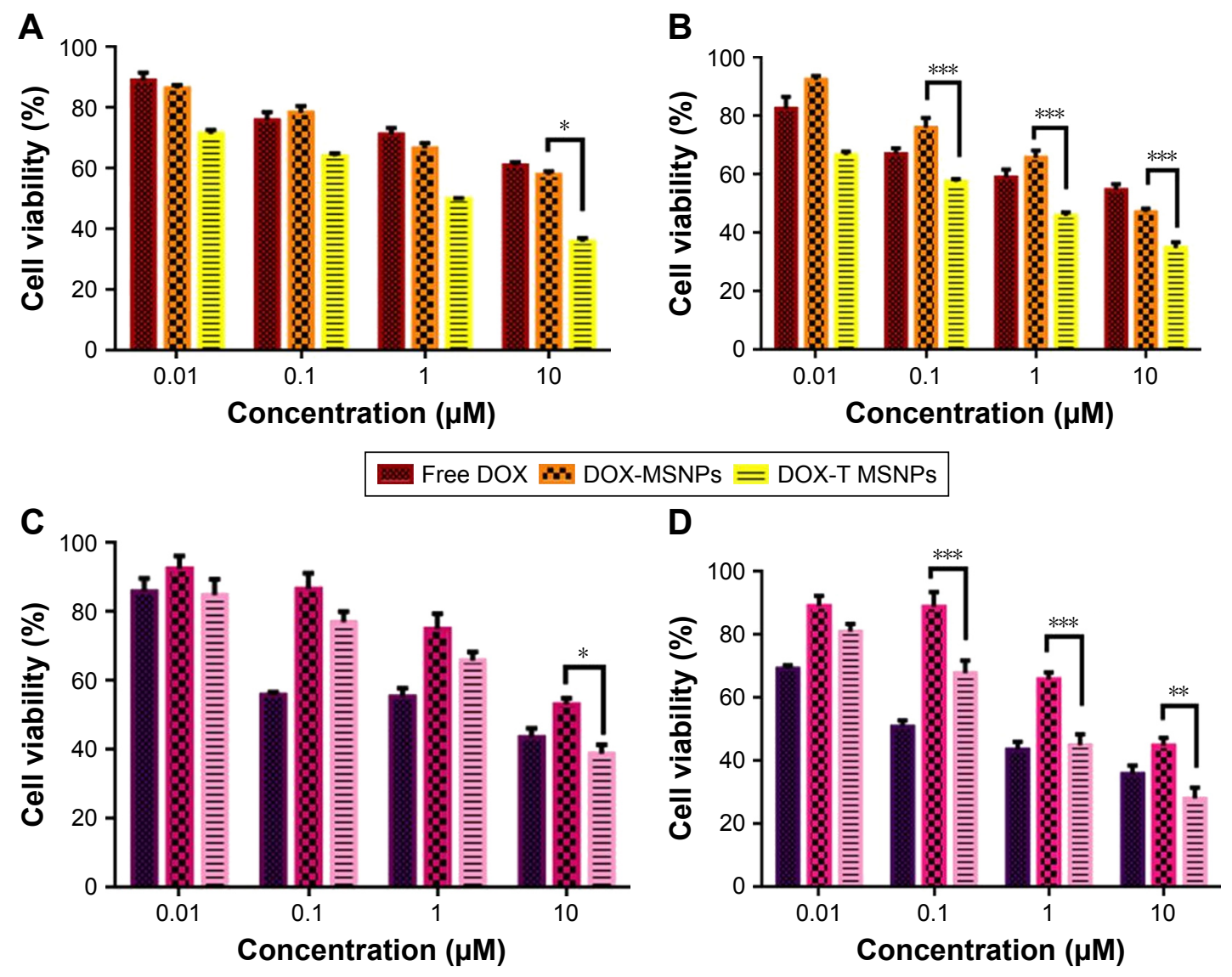

Free DOX WOX-MSNPs $=$ DOX-T MSNPs

Figure 4 Cytotoxicity assay.

Notes: Cytotoxicity assay in MCF-7 cells at (A) 24 hours and (B) 48 hours and in LNCaP cells at (C) 24 hours and (D) 48 hours at $37^{\circ} \mathrm{C}$. Data are presented as mean \pm SD. $\mathrm{n}=3 . * \mathrm{p}<0.05, * * \mathrm{p}<0.01$, $* * * \mathrm{p}<0.001$.

Abbreviations: DOX, doxorubicin; MSNPs, mesoporous silica nanoparticles. 
showed a significant amount of red-colored cells due to the easy uptake of free DOX as compared to green-colored nuclei in the untreated control cells (Figure 5). Also, MCF-7 cells treated with DOX-loaded targeted MSNPs showed a significant amount of red and yellow fluorescence as compared to the control cells. Few MCF-7 cells emitted orange color, indicating early apoptotic stage. Similar results were obtained in LNCaP cancer cells (Figure 5). To further confirm the results obtained by $\mathrm{AO} / \mathrm{EB}$ staining and quantitate the percentage of treatment-induced apoptosis, Annexin V-FITC/PI staining assay was performed using flow cytometry. As shown in Figure 6, most of the cells were observed in the early and late apoptotic phases $(\sim 43 \%)$ in the case of cells treated with free DOX. The DOX-loaded targeted MSNPs exhibited significantly higher apoptotic cells $(\sim 36 \%)$ as compared to DOX-loaded bare NPs $(\sim 13 \%)$. The results suggested that treatment with DOX-loaded targeted MSNPs induced higher apoptosis than DOX-loaded bare NPs in MCF-7 cells.

\section{Discussion}

The ability to target therapeutic agents specifically to cancer cells, thereby reducing nonspecific toxicity, determines the success of a new nanocarrier. ${ }^{26}$ In this work, MSNPs were synthesized by one-pot synthesis method, modified with targeting ligand triptorelin, and characterized for targeted drug delivery toward GnRH-overexpressing cancer cells. The synthesized MSNPs were characterized based on their size and charge. The synthesized MSNPs were $113 \mathrm{~nm}$ (hydrodynamic radius) and $55 \mathrm{~nm}$ (actual) in size, as determined by Nanosight and TEM, respectively. A previous study suggests that NPs accumulate in the tumor site due to its leaky vasculature which has fenestrae of $200 \mathrm{~nm} .{ }^{27}$ Therefore, in our study, we ensured that the synthesized MSNPs, with or without PEG and triptorelin conjugation, were smaller than $200 \mathrm{~nm}$. A negative charge on the surface of the targeted MSNPs (-17.94 mV) further ensured reduced nonspecific uptake of MSNPs by the cells. Also, the porous nature of the MSNPs enhanced entrapment of the cargo. ${ }^{28}$

In our study, targeted MSNPs conjugated with triptorelin showed a higher loading of DOX, which could be attributed to the presence of PEG and ligand on the surface of MSNPs. It has been reported that in addition to drug loading, its premature release from the NPs can also be controlled by sealing the pores of MSNPs at the periphery using conjugation of ligand or large molecules and clusters. ${ }^{29}$

Internalization studies showed higher uptake of DOXloaded targeted MSNPs as compared to DOX-loaded bare MSNPs in both the cell lines. The cells treated with DOX- loaded targeted MSNPs showed higher DOX fluorescence due to the presence of triptorelin, which facilitated GnRH receptor-mediated enhanced uptake in these cells. GnRHoverexpressing property of the cells was precisely exploited to deliver DOX-loaded targeted MSNPs, which resulted in increased uptake. However, bare MSNPs devoid of ligand (and with negative surface charge) hindered their uptake. Cells treated with free DOX showed a very high intensity of red fluorescence in the nucleus. This could be due to the huge accumulation of DOX as it can easily enter the nucleus intercalating the nucleic acids.

The cytotoxicity assay was performed in the breast as well as prostate cancer cell lines and significant cytotoxicity was observed due to free DOX and DOX-loaded MSNPs in a concentration-dependent manner. DOX-loaded targeted MSNPs showed higher toxicity than DOX-loaded bare MSNPs, which could be attributed to their higher uptake through receptor-mediated endocytosis. Thus, the cytotoxicity assay confirmed the results of cellular uptake studies, suggesting that the internalized DOX-loaded targeted MSNPs have delivered the drug, which was confirmed through the percentage viability of the cancer cells.

$\mathrm{AO} / \mathrm{EB}$ staining allows detection of apoptotic cell death through morphological changes, namely, necrotic and apoptotic stages including early and late phases. AO is a dye that stains viable and damaged cells as they are membrane permeable, while EB dye only stains dead cells wherein the membrane is damaged. $\mathrm{AO} / \mathrm{EB}$ dual staining assay was performed on MCF-7 and LNCaP cancer cells, wherein the cells were treated with free DOX, DOX-loaded targeted MSNPs, and DOX-loaded bare MSNPs for 24 hours and staining was performed. DOX-loaded targeted MSNPs showed numerous greenish-yellow cells with granulated nuclei, suggesting that these were undergoing apoptosis. Also, dead cells showed red fluorescence due to intercalation of EB in their DNA. Conversely, most of the cells treated with DOX-loaded bare MSNPs appeared green with normal nuclei morphology, suggesting that they were alive owing to the lower cellular uptake of bare MSNPs. This study has helped in determination of apoptosis in cells treated with DOX-loaded targeted NPs and also has made an accurate distinction between different stages of apoptosis and induced morphological changes ${ }^{30}$ Further, cells stained with Annexin V-FITC and PI demonstrated early and late apoptosis. Phosphatidylserine present in the inner membrane of cells gets translocated to the outer plasma membrane surface after initiation of apoptosis. While Annexin V conjugated to a fluorescent dye binds efficiently to the exposed phosphatidylserine, damaged 

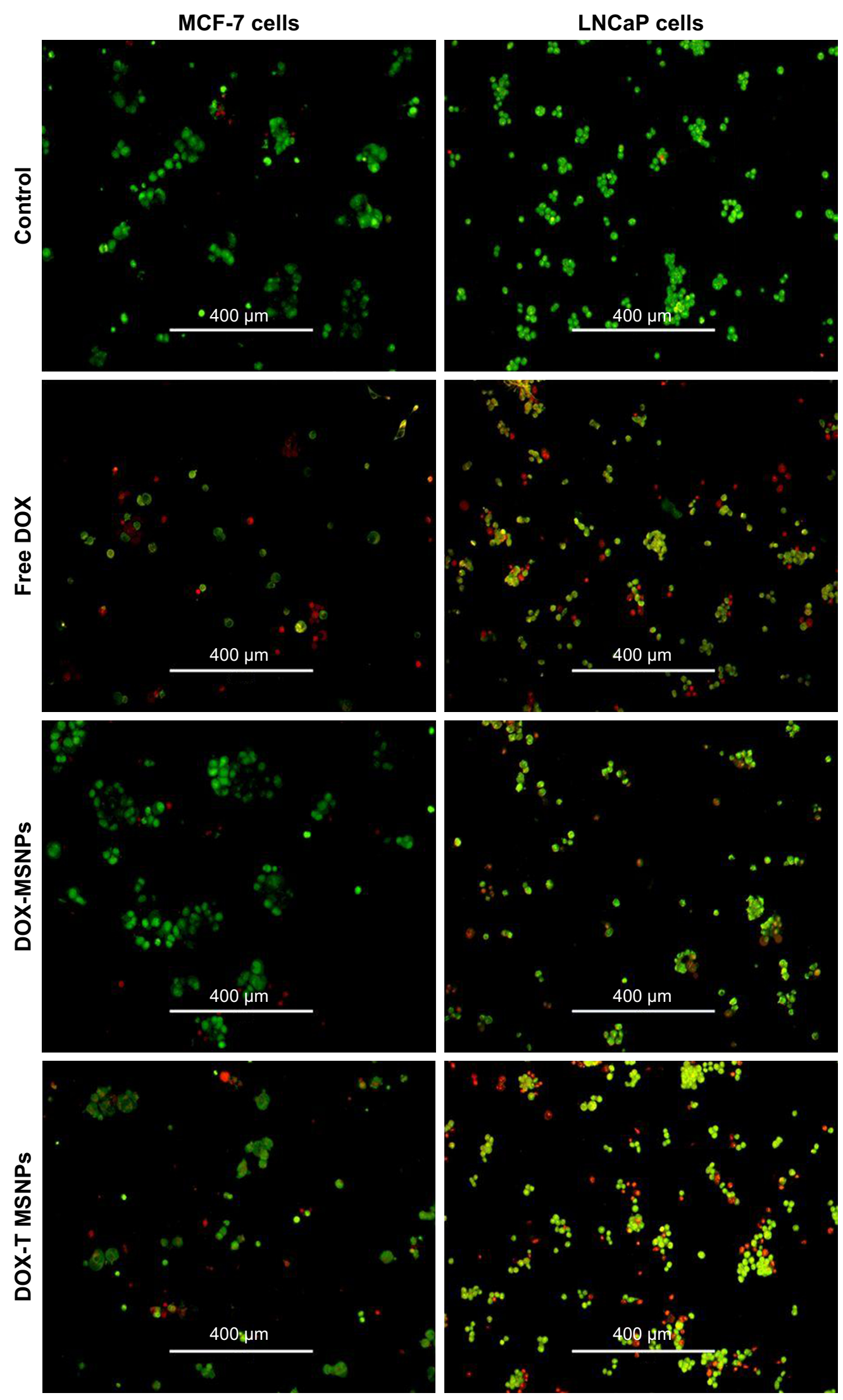

Figure 5 AO/EB dual staining in MCF-7 and LNCaP cells after treatment with free DOX, DOX-loaded bare MSNPs (DOX-MSNPs), and DOX-loaded targeted MSNPs (DOX-T MSNPs).

Abbreviations: AO, acridine orange; DOX, doxorubicin; EB, ethidium bromide; MSNPs, mesoporous silica nanoparticles. 
A

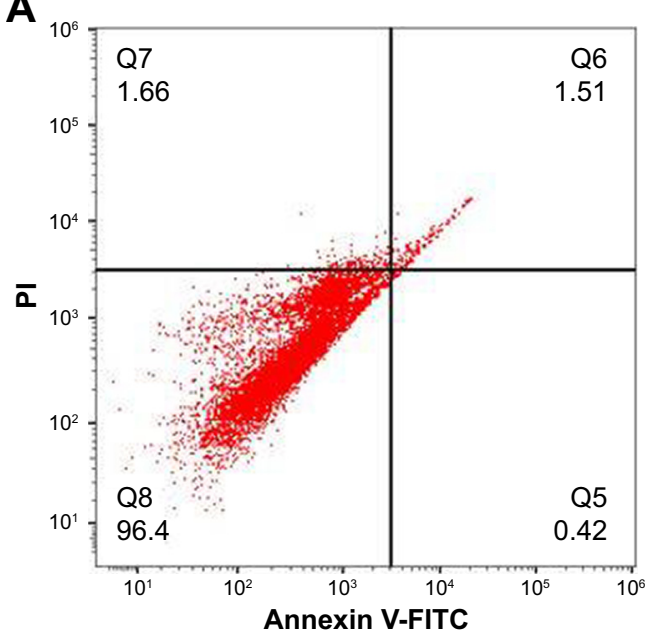

C

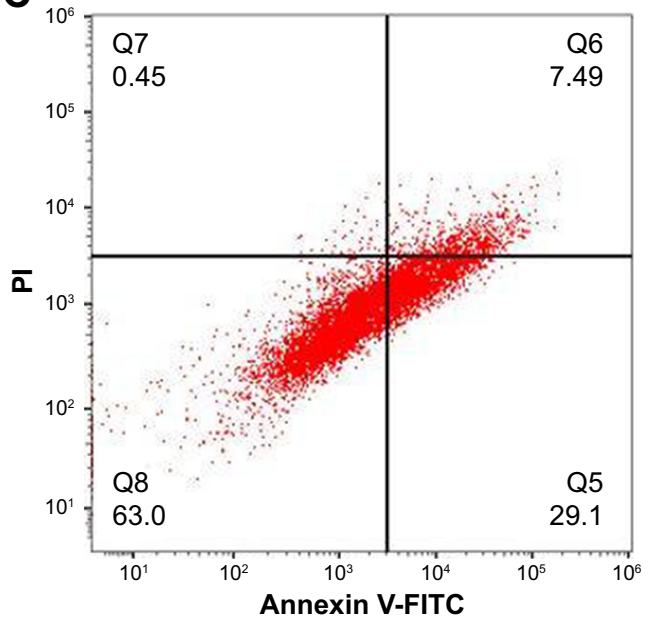

B

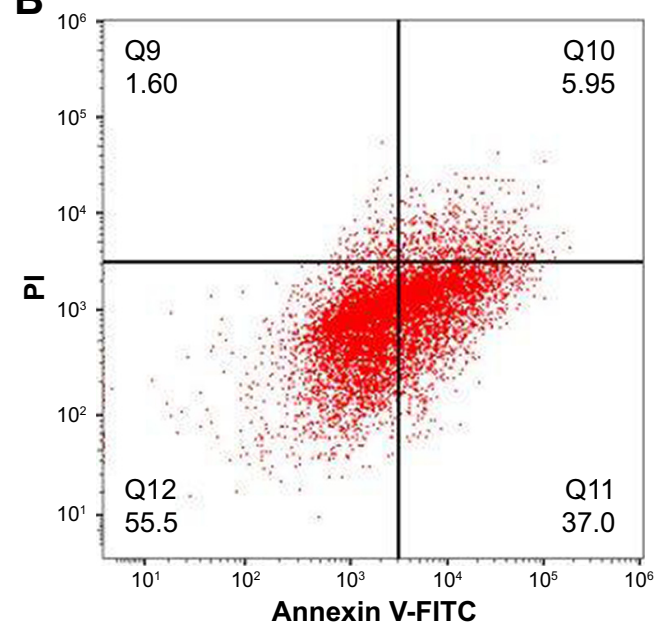

D

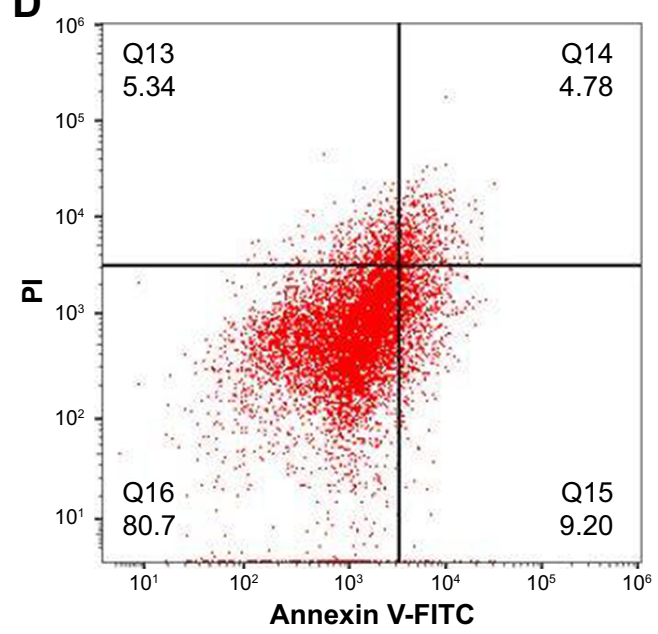

Figure 6 Apoptosis assay.

Note: Flow cytometer analysis of (A) untreated control cells, (B) free DOX-treated cells, (C) DOX-loaded targeted MSNP-treated cells, and (D) cells treated with DOXloaded bare MSNPs.

Abbreviations: DOX, doxorubicin; FITC, fluorescein isothiocyanate; MSNPs, mesoporous silica nanoparticles; PI, propidium iodide.

and dead cells are stained by PI. This assay thus confirmed the mode, phases, and percentage of apoptosis induced by DOX-loaded MSNPs. ${ }^{31}$

\section{Conclusion}

We developed MSNPs modified with PEG-triptorelin for specific targeted DOX delivery in GnRH receptor-overexpressing cancer cells. Higher loading of DOX was observed on targeted MSNPs than bare MSNPs, which were efficiently internalized in MCF-7 and LNCaP cancer cells. DOX-loaded targeted MSNPs showed significant cytotoxicity compared to DOX-loaded bare MSNPs. Moreover, apoptosis studies showed the mode of action and their early/late apoptotic stages. In conclusion, the developed multifunctional nanocarriers possess desirable attributes, such as efficient drug loading and improved targeted delivery to the cancer cells. Given these attributes, it would be worthwhile pursuing further work on different cancer models in vivo and assessing the efficacy of targeted drug delivery using MSNPs modified with PEG-triptorelin.

\section{Acknowledgment}

Dr Virendra Gajbhiye acknowledges INSPIRE Faculty Award (IFA/2013/LSBM-78) received from the Department of Science and Technology, Government of India, New Delhi, India.

\section{Disclosure}

The authors report no conflicts of interest in this work.

\section{References}

1. Roser M, Ritchie H. Cancer. Published online at OurWorldInData.org. Available from: https://ourworldindata.org/cancer. Accessed October 12, 2018. 
2. GBD. Causes of death collaborators. Lancet. 2016;2017(390):1151.

3. Zhang D, Xu Q, Wang N, et al. A complex micellar system co-delivering curcumin with doxorubicin against cardiotoxicity and tumor growth. Int J Nanomedicine. 2018;13(13):4549-4561.

4. Xu P, Zuo H, Chen B, et al. Doxorubicin-loaded platelets as a smart drug delivery system: an improved therapy for lymphoma. Sci Rep. 2017; 7:42632.

5. Ditto AJ, Shah KN, Robishaw NK, Panzner MJ, Youngs WJ, Yun YH. The interactions between L-tyrosine based nanoparticles decorated with folic acid and cervical cancer cells under physiological flow. Mol Pharm. 2012;9(11):3089-3098.

6. De Jong WH, Borm PJ. Drug delivery and nanoparticles: applications and hazards. Int J Nanomedicine. 2008;3(2):133-149.

7. Argyo C, Weiss V, Bräuchle C, Bein T. Multifunctional mesoporous silica nanoparticles as a universal platform for drug delivery. Chem Mater. 2014;26(1):435-451.

8. Mamaeva V, Sahlgren C, Lindén M. Mesoporous silica nanoparticles in medicine - recent advances. Adv Drug Deliv Rev. 2013;65(5):689-702.

9. Bahrami B, Hojjat-Farsangi M, Mohammadi H, et al. Nanoparticles and targeted drug delivery in cancer therapy. Immunol Lett. 2017;190: 64-83.

10. Yousefpour P, Atyabi F, Vasheghani-Farahani E, Movahedi AA, Dinarvand R. Targeted delivery of doxorubicin-utilizing chitosan nanoparticles surface-functionalized with anti-Her2 trastuzumab. Int J Nanomedicine. 2011;6:1977-1990.

11. Dostalova S, Cerna T, Hynek D, et al. Site-directed conjugation of antibodies to apoferritin nanocarrier for targeted drug delivery to prostate cancer cells. ACS Appl Mater Interfaces. 2016;8(23):14430-14441.

12. Wang $\mathrm{C}$, Wang $\mathrm{X}$, Zhong $\mathrm{T}$, et al. The antitumor activity of tumorhoming peptide-modified thermosensitive liposomes containing doxorubicin on MCF-7/ADR: in vitro and in vivo. Int J Nanomedicine. 2015; 10:2229-2248.

13. Liu Y, Chen Q, Xu M, et al. Single peptide ligand-functionalized uniform hollow mesoporous silica nanoparticles achieving dual-targeting drug delivery to tumor cells and angiogenic blood vessel cells. Int $J$ Nanomedicine. 2015;10:1855-1867.

14. Han Y, Zhang Y, Li D, Chen Y, Sun J, Kong F. Transferrin-modified nanostructured lipid carriers as multifunctional nanomedicine for codelivery of DNA and doxorubicin. Int J Nanomedicine. 2014;9:4107-4116.

15. Nukolova NV, Oberoi HS, Zhao Y, Chekhonin VP, Kabanov AV, Bronich TK. LHRH-targeted nanogels as a delivery system for cisplatin to ovarian cancer. Mol Pharm. 2013;10(10):3913-3921.

16. Limonta P, Montagnani Marelli M, Mai S, Motta M, Martini L, Moretti RM. GnRH receptors in cancer: from cell biology to novel targeted therapeutic strategies. Endocr Rev. 2012;33(5):784-811.

17. Schally AV, Arimura A, Kastin AJ, et al. Gonadotropin-releasing hormone: one polypeptide regulates secretion of luteinizing and folliclestimulating hormones. Science. 1971;173(4001):1036-1038.
18. Kakar SS, Jin H, Hong B, Eaton JW, Kang KA. LHRH receptor targeted therapy for breast cancer. Adv Exp Med Biol. 2008;614:285-296.

19. Kumar P, Tambe P, Paknikar KM, Gajbhiye V. Folate/ $N$-acetyl glucosamine conjugated mesoporous silica nanoparticles for targeting breast cancer cells: a comparative study. Colloids Surf B Biointerfaces. 2017;156:203-212.

20. Tambe P, Kumar P, Karpe YA, Paknikar KM, Gajbhiye V. Triptorelin tethered multifunctional PAMAM-histidine-PEG nanoconstructs enable specific targeting and efficient gene silencing in LHRH overexpressing cancer cells. ACS Appl Mater Interfaces. 2017;9(41):35562-35573.

21. Chatterjee B, Ghoshal A, Chattopadhyay A, Ghosh SS. dGTP-templated luminescent gold nanocluster-based composite nanoparticles for cancer theranostics. ACS Biomater Sci Eng. 2018;4(3):1005-1012.

22. Zheng X, Wang C, Dai J, Shi W, Yan Y. Design of mesoporous silica hybrid materials as sorbents for the selective recovery of rare earth metals. J Mater Chem A Mater. 2015;3(19):10327-10335.

23. Varshosaz J, Jahanian-Najafabadi A, Ghazzavi J. Luteinizing hormonereleasing hormone targeted poly(methyl vinyl ether maleic acid) nanoparticles for doxorubicin delivery to MCF-7 breast cancer cells. IET Nanobiotechnol. 2016;10(4):206-214.

24. Fraschini C, Jalabert M, Prud'homme RE. Physical characterization of blends of poly(D-lactide) and LHRH (a leuprolide decapeptide analog). Biomacromolecules. 2005;6(6):3112-3118.

25. Mohan P, Rapoport N. Doxorubicin as a molecular nanotheranostic agent: effect of doxorubicin encapsulation in micelles or nanoemulsions on the ultrasound-mediated intracellular delivery and nuclear trafficking. Mol Pharm. 2010;7(6):1959-1973.

26. Kumar P, Tambe P, Paknikar KM, Gajbhiye V. Mesoporous silica nanoparticles as cutting-edge theranostics: Advancement from merely a carrier to tailor-made smart delivery platform. J Control Release. 2018;287:35-37.

27. Park SM, Aalipour A, Vermesh O, Yu JH, Gambhir SS. Towards clinically translatable in vivo nanodiagnostics. Nat Rev Mater. 2017;2(5): 17014-17020.

28. Lehman SE, Mudunkotuwa IA, Grassian VH, Larsen SC. Nano-bio interactions of porous and nonporous silica nanoparticles of varied surface chemistry: a structural, kinetic, and thermodynamic study of protein adsorption from RPMI culture medium. Langmuir. 2016;32(3): 731-742.

29. Cauda V, Engelke H, Sauer A, et al. Colchicine-loaded lipid bilayer-coated $50 \mathrm{~nm}$ mesoporous nanoparticles efficiently induce microtubule depolymerization upon cell uptake. Nano Lett. 2010;10(7):2484-2492.

30. Liu K, Liu PC, Liu R, Wu X. Dual AO/EB staining to detect apoptosis in osteosarcoma cells compared with flow cytometry. Med Sci Monit Basic Res. 2015;21:15-20.

31. Kumar P, Paknikar KM, Gajbhiye V. A robust pH-sensitive unimolecular dendritic nanocarrier that enables targeted anti-cancer drug delivery via GLUT transporters. Colloids Surf B Biointerfaces. 2018;171:437-444.
International Journal of Nanomedicine

\section{Publish your work in this journal}

The International Journal of Nanomedicine is an international, peerreviewed journal focusing on the application of nanotechnology in diagnostics, therapeutics, and drug delivery systems throughout the biomedical field. This journal is indexed on PubMed Central, MedLine, CAS, SciSearch ${ }^{\circledR}$, Current Contents ${ }^{\circledR} /$ Clinical Medicine,

\section{Dovepress}

Journal Citation Reports/Science Edition, EMBase, Scopus and the Elsevier Bibliographic databases. The manuscript management system is completely online and includes a very quick and fair peer-review system, which is all easy to use. Visit http://www.dovepress.com/ testimonials.php to read real quotes from published authors. 